\section{Juego, ingenio y emoción: otra forma de aprender matemática}

\author{
Mariel Lovatto \\ ma.Ivtto@gmail.com
}

Claudia Zanabria

claudia.m.zanabria@gmail.com

María Cecilia Municoy

mcmunicoy@live.com

Belquis Alaniz

belquisnalaniz@gmail.com
Agustina Huespe

aguhuespe16@gmail.com

Docentes investigadoras de la

Facultad de Ciencias Económicas. Universidad Nacional del Litoral, Argentina.
Integración de la docencia y la extensión /

Intervenciones

RECEPCIÓN: 24/06/16

ACEPTACIÓN FINAL: 31/08/16

\section{Resumen}

Enmarcado en la III Convocatoria a Prácticas de Educación Experiencial, este proyecto tuvo como eje temático el proceso de enseñanza de la matemática desde la mirada de la "motivación", perspectiva ésta que implica el uso de estrategias que incorporan fundamentalmente un corte lúdico además de herramientas tecnológicas y comunicativas. Pensado y ejecutado por docentes investigadores de la Facultad de Ciencias Económicas de la Universidad Nacional del Litoral y estudiantes que cursaban materias correspondientes al área Matemática. Fue construido con base en los aportes de distintos autores que han valorado la importancia del juego en el aprendizaje y han demostrado en diversos estudios que un juego produce interés, motivación, diversión, desbloqueo y gusto por estudiar matemática.

Las actividades en las Escuelas de Enseñanza Secundaria Orientadas que participaron incluyeron juegos por Internet y de mesa y permitieron verificar una muy buena relación entre la teoría indicada como soporte y la realidad.
Palabras-clave

- Motivación

- Aprendizaje

- Matemática

- Juegos

- Extensión

\section{Resumo}

Inserido na III convocatória a Práticas de Educação Experimental, este projeto teve como eixo temático o processo de ensino de matemática, desde a motivação, perspectiva que exige o uso de estratégias que incorporam fundamentalmente atividades lúdicas, além de ferramentas tecnológicas e comunicativas. A proposta foi pensada e realizada por professores pesquisadores da Faculdade de Economia da Universidade Nacional del Litoral e alunos que faziam disciplinas relacionadas à área da matemática.

O projeto foi construído com base nas contribuições de diferentes autores que têm valorado a importância do jogo na aprendizagem e demonstraram, em diversos estudos, que o jogo produz interesse, motivação, diversão, liberação e gosto pelo estudo das matemáticas.

As Atividades nas Escolas de Ensino Médio que participaram incluíram jogos on-line e de tabuleiro, e permitiram conferir uma relação muito boa entre a teoria indicada como suporte e a realidade.

Palavras-chave

- Motivação

- Aprendizagem

- Matemática

- Jogos

- Extensão

\section{Para citación de este artículo}

Lovatto, M.; Zanabria, C.; Municoy, M. C.; Alaniz, B. y

Huespe, A. (2016). Juego, ingenio y emoción: otra forma de aprender matemática. En Revista +E versión digital, (6), pp. 336-343. Santa Fe, Argentina: Ediciones UNL. 


\section{Introducción}

En los últimos años, en muchas de las escuelas secundarias de la ciudad de Santa Fe, sobre todo en las escuelas públicas, se advierten situaciones que dificultan la tarea en el aula como las condiciones académicas (carga horaria, exceso de alumnos repitentes en los cursos, sobre edad, obligatoriedad, etc.) o el ambiente sociocultural (ausencia de ayuda familiar, falta de supervisión de adultos, exceso de estímulos tecnológicos, etc.). Estas condiciones determinan que la escuela se convierta en un espacio de contención más que de aprendizaje. En consecuencia, las problemáticas que se generan influyen en la motivación por el aprendizaje en general y por el aprendizaje de la matemática en particular.

El conflicto al que se trató de aportar fue por tanto la falta de motivación como obstáculo en el aprendizaje de la matemática en los estudiantes de la Escuela de Enseñanza Secundaria Orientada (EESO).

La finalidad de la propuesta fue prestar un servicio de calidad que mitigara esta problemática, y dado que se enmarcó en el proyecto de Prácticas de Educación Experiencial (PEE). se convocaron e integraron al mismo alumnos que estaban cursando las materias Matemática Básica, Matemática para Economistas y Matemática Financiera de las carreras que se dictan en la Facultad de Ciencias Económicas (FCE) interviniendo, como guías y responsables, docentes investigadoras de las mencionadas cátedras de la Facultad, con la colaboración de una alumna ayudante de la cátedra de Matemática Básica.

Las PEE corresponden al Plan de Desarrollo Institucional de la Secretaría de Extensión de la Universidad Nacional del Litoral (UNL), que promueve la articulación entre la Universidad y el medio social en el que se encuentra inserta. Tienen como objeto promover el mejoramiento institucional de las prácticas de la enseñanza y procesos de aprendizajes, respondiendo a los fines de la educación e inscribiéndose con sentido en los contextos sociales, políticos e históricos de la vida. La educación experiencial refiere a una clase particular de aprendizaje, a una estrategia de enseñanza que tiene un enfoque holístico y cuyo fin es relacionar el aprendizaje académico con la vida real. Además con la particularidad que, desde el centro del currículo, a través de estas experiencias se promueve en los estudiantes, la indagación, la observación sistemática, la reflexión y el espíritu científico.

\section{Justificación}

El eje temático para estas PEE fue el proceso de aprendizaje de conocimientos relacionados con la matemática contando con un potente instrumento de contribución: el juego y las tareas lúdicas. Se puede acceder a esta temática por diversas puertas y en este caso se optó por la puerta "motivación", por lo que se presentaron estrategias que posibilitaran abrirla.
En este sentido, se considera a la motivación como el interés que tiene el alumno por su propio aprendizaje o por las actividades que le conducen a él. Ese interés se puede adquirir, mantener o aumentar en función de elementos intrínsecos y extrínsecos. Influyen en la motivación aspectos como el ambiente sociocultural del alumno, la confianza en sí mismo, los acervos personales, las condiciones académicas, los estilos de enseñanza y aprendizaje. En lo referente a los estilos de enseñanza, puede decirse que en las aulas prolifera el estilo de enseñanza "tradicional", con su forma "homogénea" y meramente transmisora que responde siempre con la secuencia: enseñar y ejercitar. De esto resulta que las matemáticas que se enseñan en el sistema escolar no son las mismas que se utilizan en el hacer cotidiano, se aprenden de forma descontextualizada, sin relación con hechos o situaciones concretas. Si la intención es "motivar" a los alumnos, su enseñanza debe estar definida por sus usos sociales y no solamente en la epistemología de esta ciencia.

En lo que respecta a los estilos de aprendizaje, el área de Matemática de la FCE propicia en sus estudiantes el aprendizaje de la matemática como un lenguaje. Este estilo de aprendizaje trasciende al contenido y favorece la comprensión tanto de la realidad científica como de la cotidiana.

A lo largo de la historia, distintas investigaciones coinciden en considerar que la falta de motivación es un obstáculo en el aprendizaje de la matemática. En este sentido, Font (1994) afirmaba que cualquier análisis del aprendizaje de las matemáticas debe considerar la motivación, la actitud hacia las matemáticas será diferente según tenga el estudiante un patrón motivacional positivo o negativo. Es de esperar un patrón positivo, que hace que frente a una dificultad el estudiante reaccione analizándola, buscando estrategias nuevas, preguntando al profesor, etcétera.

Del Informe PISA 2003 (OCDE, 2005) puede concluirse que la motivación influye en los alumnos de escuela secundaria en cuanto a la adopción de estrategias de aprendizaje eficaces.

Para desprenderse de lo "tradicional", acorde con el nivel de educación formal donde se realizó la intervención, se utilizó el juego para desarrollar contenidos y competencias, como una forma diferente de abordar el aprendizaje y la enseñanza de la matemática. ¿Por qué el juego para motivar el aprendizaje y la enseñanza de las matemáticas? ¿Por qué el juego en el marco de la educación experiencial como otra forma de aprender?

En cuanto a la primera pregunta, distintos autores han valorado su importancia en el aprendizaje ya que produce entusiasmo, interés, diversión, desbloqueo y gusto por estudiar matemáticas. Adela Salvador (2013) sostiene que un juego bien elegido puede servir para introducir un tema, ayudar a comprender mejor los conceptos o procesos, afianzar los ya adquiridos, conseguir destreza en algún algoritmo, reforzar automatismos y consolidar un contenido. El juego es una actividad aceptada con facilidad por los estudiantes. 


\section{6}

el proyecto tuvo como finalidad

brindar un servicio de calidad que

permita mitigar el problema de la

falta de motivación en el aprendizaje

de la matemática en estudiantes

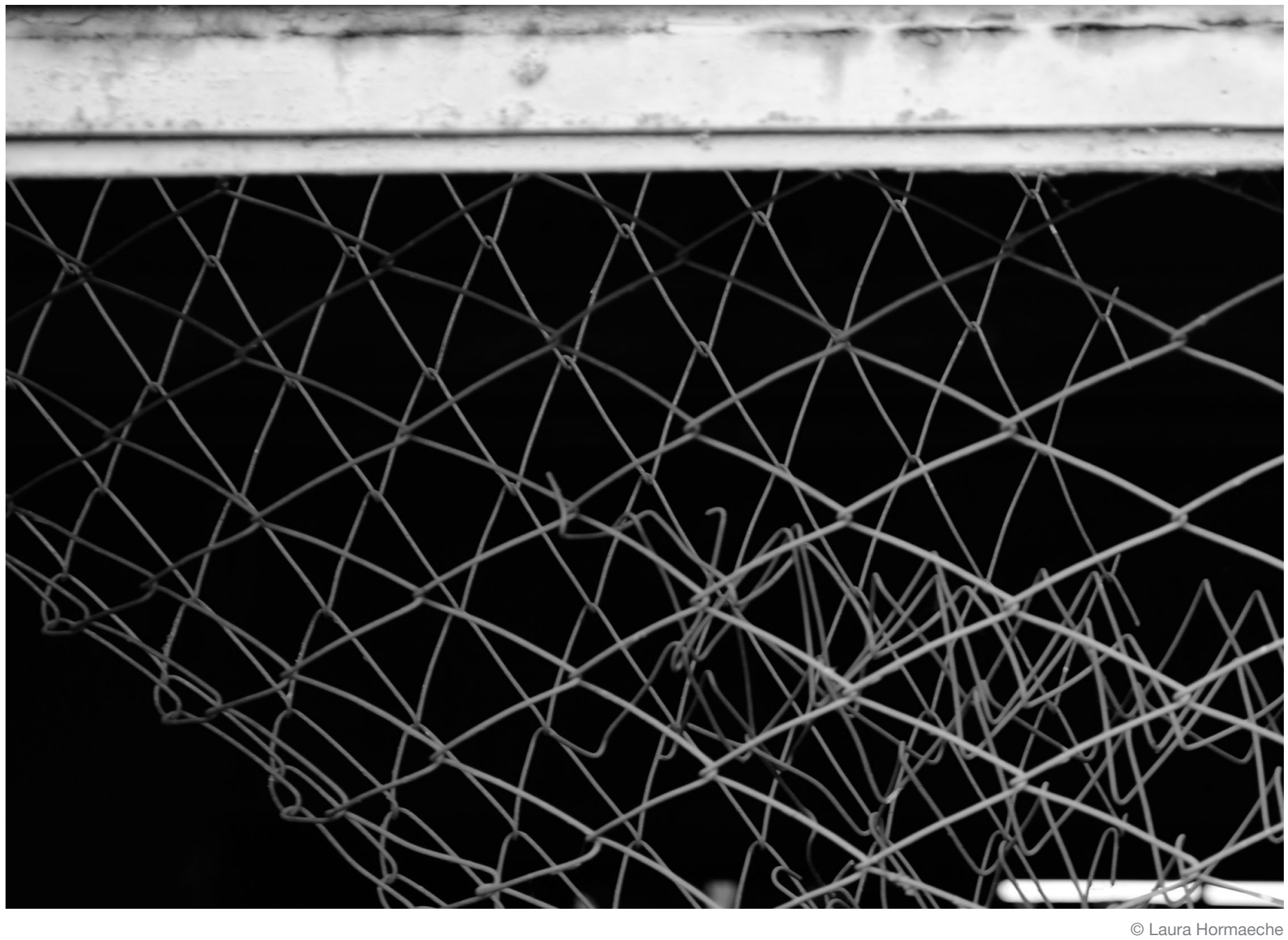


Jugar hace que se estimule el desarrollo social y se ven favorecidas las relaciones con sus compañeros de aula, la empatía, la cooperación y el trabajo en equipo, la aceptación y seguimiento de normas y la discusión de ideas. Los alumnos deben hablar, compartir, para después comprobar y explicar.

En el terreno de la matemática se observa el paralelismo entre las fases de los juegos de estrategia y la resolución de problemas. Este paralelismo fomenta el descubrimiento de procesos heurísticos en los alumnos (Corbalán, 1996; Gairín 2006; Hernández 2010). Despiertan en los alumnos la necesidad de hacer preguntas, elaborar estrategias, hacer deducciones y llevados a cabo en grupos, puede ser estimulantes, intrigantes, divertidos y gratificantes. Desarrollan capacidades cognitivas en los tres niveles de representación: enactivo, icónico y simbólico. Requieren esfuerzo, rigor, atención, memoria y estimulan la imaginación (Alsina 2007).

En cuanto a enmarcar esta experiencia en la educación experiencial, surgió de entender que, como dice Camilloni (2013), hay que proponerle al alumno universitario actividades donde, en conexión con la práctica, se le demande que ponga a prueba sus habilidades y conceptos teóricos en una situación auténtica, real, (en este caso, la planificación y puesta en práctica de clases). Estimularlo para que juzgue las consecuencias de sus acciones, enriquezca sus conocimientos y habilidades, identifique nuevos problemas y determine prioridades en cuanto a las urgencias de su solución. Adherimos además a la reflexión de esta autora, quien sostiene que cuando el aprendizaje es experiencial, quien aprende, tanto el estudiante de la FCE como el de la escuela secundaria, lo hace en las condiciones sociales de la utilización auténtica de los conocimientos. Los estudiantes aprenden en un ámbito que tiene como objetivo el logro simultáneo de fines comunitarios y educativos. El alumno aprende a usar los conocimientos en el trabajo. Este trabajo es tal que le otorga derechos y responsabilidades y promueve la adopción de decisiones autónomas.

Esta experiencia se incluye en la modalidad de aprendizaje-servicio, dado que los estudiantes utilizan sus conocimientos y habilidades académicas para dar satisfacción a necesidades reales de la sociedad y responder a los requerimientos manifestados por la comunidad. Esta estrategia de enseñanza contribuye además a la formación ética de los estudiantes universitarios, porque ellos deben comprometerse con los valores del proyecto en el que participa, de la universidad y de los grupos a los que está destinada su acción.

\section{3. Ámbito de aplicación}

La matemática es un área disciplinar obligatoria durante toda la EESO. Para el primer y segundo años, la materia cuenta con cinco horas semanales, cuatro en el tercer año, y cuarto y quinto años tienen, dependiendo de la orientación, tres o cuatro horas semanales. Los contenidos comunes incluyen conjuntos numéricos, álgebra, geometría, funciones y gráficas y estadística y probabilidad. Las escuelas con las que se trabajó tienen una zona de influencia que se extiende a barrios colindantes cuyos habitantes tienen un nivel económico medio-bajo y con un nivel de estudios medios y superiores inferior a los que se registran en otras zonas de la ciudad. ${ }^{1}$ Estas escuelas fueron elegidas teniendo en cuenta las necesidades y dificultades puestas de manifiesto por docentes que dictan clases en ellas, que por diferentes motivos tienen una relación cercana a esta Unidad Académica y por tanto fueron nuestros referentes. $Y$ si bien es sabido que tales dificultades son compartidas por numerosos establecimientos de nuestra ciudad, nuestra decisión de limitar el proyecto a solo tres instituciones se fundó en cuestiones de organización, de tiempo áulico para llevar a cabo las experiencias y posibilidades del equipo docente.

\section{Propósitos del Proyecto de Educación Experiencial}

El proyecto tuvo como finalidad brindar un servicio de calidad que permita mitigar el problema de la falta de motivación en el aprendizaje de la matemática en estudiantes de ESSO de la ciudad de Santa Fe a través de propuestas de actividades lúdicas. De los alumnos de la FCE, acompañados por sus docentes, se esperaba que, empleando la heurística y el lenguaje matemático generaran estrategias de aprendizaje de la matemática adecuadas para propiciar el interés y la motivación de los estudiantes de las instituciones educativas involucradas en este proyecto. En este marco, los estudiantes de la FCE realizaron prácticas que enlazaron diversos niveles de interacción e intervención en las instituciones educativas participantes de modo que significara un aporte a su formación académica y profesional. Estas estrategias contemplaron la incorporación de herramientas tecnológicas y comunicativas y tuvieron fundamentalmente un corte lúdico.

El proyecto pretendió que los estudiantes de la FCE pudieran:

- Valorar el aprendizaje de la matemática como lenguaje.

- Transmitir la idea de que se puede jugar y aprender.

- Favorecer, mediante estrategias que despierten su motivación, la comprensión de conceptos matemáticos.
1) Las escuelas son: EESO $n^{\circ} 256$

General Juan Bautista Bustos; EESO

$\mathrm{n}^{\circ} 264$ Constituyentes; y EESO y Mo- dalidad Técnica Particular Incorporada $n^{\circ} 2025$ Ceferino Namuncurá, de la ciudad de Santa Fe. 
- Poner en evidencia la importancia de los procesos de pensamiento de la matemática.

- Reflexionar sobre las actividades didácticas desarrolladas para construir sus propias ideas matemáticas.

- Aplicar lo "enseñado" en su propio aprendizaje.

Por su parte, de los estudiantes de las escuelas se esperó que lograran:

- Romper el bloqueo y sortear el rechazo que sienten muchos alumnos al aprender matemática.

- Experimentar placer al jugar y aprender.

- Valorar el juego como metodología de aprendizaje.

- Incorporar las técnicas de los juegos para resolver problemas matemáticos, analizando fases, estrategias heurísticas y buscando un modelo matemático.

- Valorar el trabajo solidario y en cooperación para la búsqueda de mejores soluciones.

\section{Desarrollo del proyecto}

Los contenidos curriculares relacionados son: funciones, gráficas, resolución de ecuaciones y sistemas de ecuaciones, trabajo con expresiones algebraicas, resolución de problemas, notaciones simbólicas (significado).

Las actividades planificadas desarrolladas durante el segundo semestre de 2015 se organizaron en distintas etapas y espacios. Una primera etapa de organización se llevó a cabo en la FCE. En esta etapa, además de darles a conocer la propuesta a los alumnos que aceptaron participar del proyecto, se brindó información sobre las características y peculiaridades de cada institución involucrada. En las reuniones siguientes estudiantes y docentes se abocaron a preparar actividades que propiciaran el uso del lenguaje matemático con finalidad diagnóstica, se organizaron, elaboraron y seleccionaron juegos, tanto digitales como de mesa, teniendo en cuenta los contenidos curriculares a abordar, las estrategias de aprendizaje que incluyeran actividades lúdicas, como así también el uso de textos no matemáticos que aportaran al aprendizaje del lenguaje matemático. Finalmente, se definieron fechas y horarios en las instituciones escolares invitadas a participar.

En la segunda, tercera, cuarta y quinta etapa, desarrolladas simultáneamente en las escuelas y en la FCE, se hicieron las actividades de evaluación diagnóstica que aportaron información sobre lo que piensan o sienten los alumnos sobre el aprendizaje de la matemática. En función de ello, se ajustaron las actividades planificadas, se realizaron actividades lúdicas y se hizo una puesta en común para analizar soluciones y resultados de los objetivos de aprendizajes propuestos y cumplidos.

Secuencia de juegos y actividades

\begin{tabular}{|c|c|c|}
\hline Clase & Juego-actividad & Contenido \\
\hline 1 ra. & Juego del Gran DT ${ }^{2}$ & $\begin{array}{l}\text { Introducción a la resolución de juegos } \\
\text { de ingenio relacionados con el fútbol. }\end{array}$ \\
\hline 2da. & Salvando los extraterrestres & $\begin{array}{l}\text { Introducción a la función lineal. Gráfico } \\
\text { y expresión algebraica. }\end{array}$ \\
\hline 3ra. & Algebra vs. Cockroaches ${ }^{3}$ & $\begin{array}{l}\text { Función lineal, gráficos y expresión } \\
\text { algebraica }\end{array}$ \\
\hline 4 ta. & Baraja de funciones. Trivia & $\begin{array}{l}\text { Funciones, gráficas y expresiones } \\
\text { algebraicas }\end{array}$ \\
\hline 5 ta. & Evaluación final & $\begin{array}{l}\text { Aplicación de cuestionario y papel de } \\
\text { un minuto }\end{array}$ \\
\hline
\end{tabular}

\section{Evaluación}

\subsection{Estrategias e instrumentos}

Un aspecto fundamental del proyecto fue la evaluación, que posibilitó valorar tanto a los estudiantes que participaron como a la experiencia que se generó. La recolección de información se realizó a partir de dos fuentes de registros. Una de ellas, denominada Registros 1, obtenida a través de la observación y participación en la experiencia; y otra, identificada como Registros 2, obtenida de la aplicación de instrumentos y estrategias intencionalmente diseñadas para tal fin.

Respecto de la información conseguida mediante Registros 1: Observación y participación en la experiencia, nos preguntamos: ¿cómo mirar? Y en este sentido nos apoyamos en la narrativa de Eisner (1998), quien en su obra El ojo ilustrado expresa que los sujetos vamos creando modos de mirar — que no es ver-que dependen de la experiencia y de la persona.

Sostenemos que la acción educativa no debe ser mirada solo por el ojo experto de un investigador, pues, como afirma Freire (1997), cuando profesores y alumnos aprenden o enseñan, lo hacen con sus emociones, con sus miedos, con sus pasiones, con sus cuerpos enteros, por eso la experiencia narrada de un docente o el accionar de un alumno en el aula puede constituir un instrumento
2) El Gran DT (Director Técnico) es un juego por el cual los alumnos, al resolver problemas de ingenio, ganan puntos y pueden elegir y comprar cinco de los mejores jugadores de equipos argentinos para integrar un equipo de fútbol. Se trata de un juego grupal, y el que resuelve correctamente y más rápido tiene la posibilidad de elegir primero al Dream Team.
3) Este juego aparece en Internet con el mismo nombre. Los alumnos tienen que "matar" cockroaches (cucarachas) en un sistema cartesiano, seleccionando las expresiones algebraicas de las funciones lineales sobre las que caminan los insectos. Es una página interactiva que les permite avanzar en los niveles de dificultad a medida que responden correctamente. 
privilegiado de reflexión. Motivo por el cual el conocimiento que se genera no es necesariamente transferible a otros entornos, pero sí, como dicen Gimeno Sacristán y otros (2002), debe provocar la reconstrucción de las formas de pensar, sentir y actuar de los que de alguna forma participan de una situación educacional.

Con relación a la información obtenida por Registros 2: Instrumentos y estrategias, cabe destacar que la evaluación de los estudiantes involucrados en el proyecto se realizó desglosada en tres fases, teniendo en cuenta diferentes aspectos, y se tornó un proceso de evaluación participativa que abarcó autoevaluación, coevaluación y heteroevaluación en distintos momentos de la PEE, como se describe en la siguiente tabla:

\begin{tabular}{|c|c|c|}
\hline Fases & Instrumento de evaluación & Aplicada a \\
\hline Diagnosticada & $\begin{array}{l}\text { Cuestionarios cortos sobre } \\
\text { uso del lenguaje matemático } \\
\text { en distintos contextos e } \\
\text { incorporación de reflexión sobre } \\
\text { el aprendizaje logrado o no. }\end{array}$ & $\begin{array}{l}\text { - Alumnos de la FCE } \\
\text { y de las escuelas } \\
\text { secundarias. }\end{array}$ \\
\hline Formativa & $\begin{array}{l}\text { Diario de reflexión: registro } \\
\text { de lista de dudas, actividades } \\
\text { que han presentado dificultad, } \\
\text { acontecimientos destacados, } \\
\text { lo aprendido o no en dichas } \\
\text { actividades, anécdotas, } \\
\text { reflexiones sobre la práctica, } \\
\text { aspectos claves a considerar. } \\
\text { Papel de un minuto: producción } \\
\text { de una frase u oración que, } \\
\text { finalizada la clase, resuma el } \\
\text { tema abordado. } \\
\text { Lista de cotejos: registro de } \\
\text { criterios o aspectos indicadores } \\
\text { de logro, para establecer } \\
\text { la presencia o ausencia del } \\
\text { aprendizaje alcanzado por los } \\
\text { estudiantes. }\end{array}$ & $\begin{array}{l}\text { - Alumnos de la FCE. } \\
\text { - Alumnos de las } \\
\text { escuelas secundarias. } \\
\text { - Los profesores. }\end{array}$ \\
\hline Final & $\begin{array}{l}\text { El juego de Baraja de Funciones. } \\
\text { Una indagación escrita, a modo } \\
\text { de cuestionario. } \\
\text { El papel del minuto. }\end{array}$ & $\begin{array}{l}\text { - Alumnos de las } \\
\text { escuelas secundarias. }\end{array}$ \\
\hline
\end{tabular}

\subsection{Resultados de la evaluación}

\subsubsection{De Registros 1}

La observación, participación y los registros realizados, permitieron percibir un aumento de la motivación en los alumnos de las EESO ante las propuestas de aprendizaje, en las que el juego se presentó como un dinamizador imprescindible en la adquisición de habilidades tanto afectivas como cognitivas. Una de las evidencias es la intervención y el compromiso mostrado por dichos estudiantes en las actividades propuestas. En este sentido se destaca que: algunos alumnos que en las primeras clases se mostraron ajenos a la clase se involucraron significativamente en ella, a la hora de resolver los juegos que se les planteaban, el interés por ganar los hacía involucrarse en forma directa en su aprendizaje.

En las dos últimas clases del desarrollo del proyecto, los alumnos de las EESO, formados en grupos, jugaron a la Baraja de Funciones. El objetivo del juego era avanzar a partir de la resolución de problemas en los que se involucraban las distintas representaciones de la función lineal. Otros aspectos evaluados con esta actividad fueron los que son propios de los juegos en grupo: la cooperación, el desarrollo de la expresión oral y escrita, la reflexión del trabajo para llegar a la solución, la discusión, el compartir, para después comprobar y explicar.

\subsubsection{De Registros 2}

Los alumnos de la FCE produjeron un diario que contaba con dos partes. En la primera, ellos realizaron una autoevaluación relacionada con su desempeño en el trabajo técnico-académico, diferenciando por actividad cuestiones como organización del trabajo, gestión de información, registro de datos, ejecución de acciones planificadas, entre otras; y también realizaron una mirada en torno a su desarrollo personal en el proyecto donde expresaron su compromiso y actitud frente a las actividades propuestas, lugar de aplicación, relación con pares, intervención frente a imprevistos. Los datos recabados son muy alentadores: alumnos muy satisfechos con su forma de trabajo frente a lo propuesto, comprometidos al máximo con las actividades, adaptados al entorno y con una preocupación constante por participar, opinar, actuar de manera crítica y preocupados por el logro de los objetivos y por la elaboración de propuestas de mejoras al proyecto. En la segunda parte de este diario los alumnos evaluaron el proyecto y establecieron como fortalezas la relación con el equipo de trabajo, la interacción lograda con los alumnos de las escuelas y la apreciación de la matemática desde otra perspectiva. En cuanto a sus aprendizajes, destacan, por un lado, el fortalecimiento de aquellos de contenido matemático reflejado en el correcto uso del lenguaje matemático que requerían las actividades propuestas, y por otro, la docencia como algo nuevo que implica responsabilidad, que los interpela a rever la realidad educativa en cuanto a los desafíos y al compromiso, en tanto encuentran en aquí el lugar donde aplicar lo que aprenden. Finalmente, se realizó a los alumnos de la EESO una indagación corta, individual y por escrito. Cada pregunta se elaboró para conocer la presencia de los mecanismos de articulación entre los conceptos y subconceptos atinentes a la función lineal y a la interiorización de sus diversas formas de representación. Se incluyeron cuatro ítems donde debían relacionar los siguientes registros de representación del concepto de función lineal: gráfico en el plano cartesiano, expresión algebraica, reconocimiento de pendiente y ordenada. 


\begin{tabular}{|c|c|c|c|}
\hline Item & Contenido & Correctas \% & Erróneas \% \\
\hline 1 & $\begin{array}{l}\text { Identificar elementos del concepto de } \\
\text { función a partir de la representación } \\
\text { externa: expresión algebraica. }\end{array}$ & 100 & - \\
\hline 2 & $\begin{array}{l}\text { Relacionar la representación algebraica } \\
\text { con la visualización interna del concepto. }\end{array}$ & 89,36 & 10,64 \\
\hline 3 & $\begin{array}{l}\text { Establecer la vinculación entre la } \\
\text { representación gráfica en el plano } \\
\text { cartesiano y el concepto. }\end{array}$ & 63,83 & 36,17 \\
\hline 4 & $\begin{array}{l}\text { Realizar la vinculación entre dos } \\
\text { representaciones externas del mismo } \\
\text { concepto. }\end{array}$ & 89,36 & 10,64 \\
\hline
\end{tabular}

Respecto de la evaluación de la experiencia de aprender matemática jugando, los estudiantes de las EESO debían responder con frases cortas a las preguntas: P1) ¿Qué aprendiste?, P2) ¿Qué es lo que más te gustó?, P3) ¿Qué es lo que menos te gustó? y P4) ¿Qué propondrías para un nuevo encuentro? Las respuestas dadas fueron "codificadas" para poder hacer una lectura e interpretación general, y se detallan en la siguiente tabla.

\begin{tabular}{|c|c|}
\hline \multicolumn{2}{|l|}{ Respuesta } \\
\hline 1. Ausencia de profesores & 10. Finalización del proyecto \\
\hline 2. Aprender jugando & 11. Nuevos contenidos \\
\hline 3. Juegos y actividades & 12. Ubicar coordenadas y rectas \\
\hline 4. Nuevos juegos & 13. Regalos \\
\hline 5. Resolver cuaciones & 14. Trabajar en grupo \\
\hline 6. Vínculos con profesores & 15. Juegos en internet \\
\hline 7. Nada & 16. Más clases \\
\hline 8. Todo & 17. Aprender con los profesores \\
\hline
\end{tabular}

En las filas de la siguiente tabla aparecen las preguntas realizadas y en las columnas los códigos de las respuestas más frecuentes:

\begin{tabular}{|c|c|c|c|c|c|c|c|c|c|c|c|c|c|c|c|c|c|}
\hline \multirow[t]{2}{*}{ Pregunta } & \multicolumn{17}{|c|}{ Respuesta } \\
\hline & 1 & 2 & 3 & 4 & 5 & 6 & 7 & 8 & 9 & 10 & 11 & 12 & 13 & 14 & 15 & 16 & 17 \\
\hline & - & - & - & - & - & - & - & - & - & - & - & - & - & - & - & - & - \\
\hline P1 & & 7 & 3 & & 6 & & 3 & 5 & 12 & & 2 & 7 & & 5 & 3 & & \\
\hline & - & - & - & - & - & - & - & - & - & - & - & - & - & - & - & - & $\overline{1}$ \\
\hline P2 & & 2 & 20 & & 5 & 2 & 1 & 3 & 8 & & & & 1 & 2 & 10 & & 4 \\
\hline & - & 一 & - & - & - & - & - & - & - & - & - & - & - & - & - & - & - \\
\hline P3 & 1 & & 4 & & & & 14 & & & 4 & & & & & 6 & & \\
\hline & - & 一 & & - & - & - & - & - & - & - & - & 一 & - & 一 & - & - & - \\
\hline P4 & & 6 & & 11 & & & & & & & 8 & & & 4 & & 8 & \\
\hline
\end{tabular}

Estas respuestas de los estudiantes secundarios dan cuenta de que ellos disfrutaron de la experiencia, de que jugando aprendieron la función lineal, sus gráficos y expresiones algebraicas. Se sintieron a gusto con el trabajo en grupo y demandaron que se les ofrezcan nuevos juegos para seguir aprendiendo otros contenidos matemáticos.

\section{Conclusiones}

A partir de los resultados anteriores, se evidencia un aumento de la motivación y del interés hacia el estudio de la matemática en el aula con la implementación de esta metodología. Los estudiantes afirman que aprender matemáticas jugando les resulta interesante y divertido. También reconocen que el uso de juegos les facilitó la comprensión del concepto de función lineal y el trabajo en grupos les permitió mejorar su aprendizaje. Asimismo, se pusieron de manifiesto valores como la solidaridad, el compromiso, el respeto, la tolerancia, la independencia, el pensamiento crítico, la honestidad, la generosidad, la humildad y la laboriosidad, tanto de parte de los estudiantes de la FCE como de parte de los estudiantes de la EESO.

Los alumnos de la FCE integrantes del proyecto trabajaron totalmente en forma colaborativa, tanto en la toma de decisiones como en la elección de las actividades, con una participación y compromiso permanente frente al aprendizaje propio y de los estudiantes de las escuelas secundarias.

La experiencia tuvo además varios aspectos positivos, a saber: el vínculo creado, de los estudiantes de la FCE entre sí y con los alumnos de las EESO que generó la confianza necesaria para que pudieran preguntar, indagar, cuestionar, de tal manera que se los veía aprendiendo y disfrutando a la vez. Por otra parte, la experiencia les posibilitó a los alumnos de la FCE reconocer sus propias fortalezas y debilidades frente al aprendizaje. 


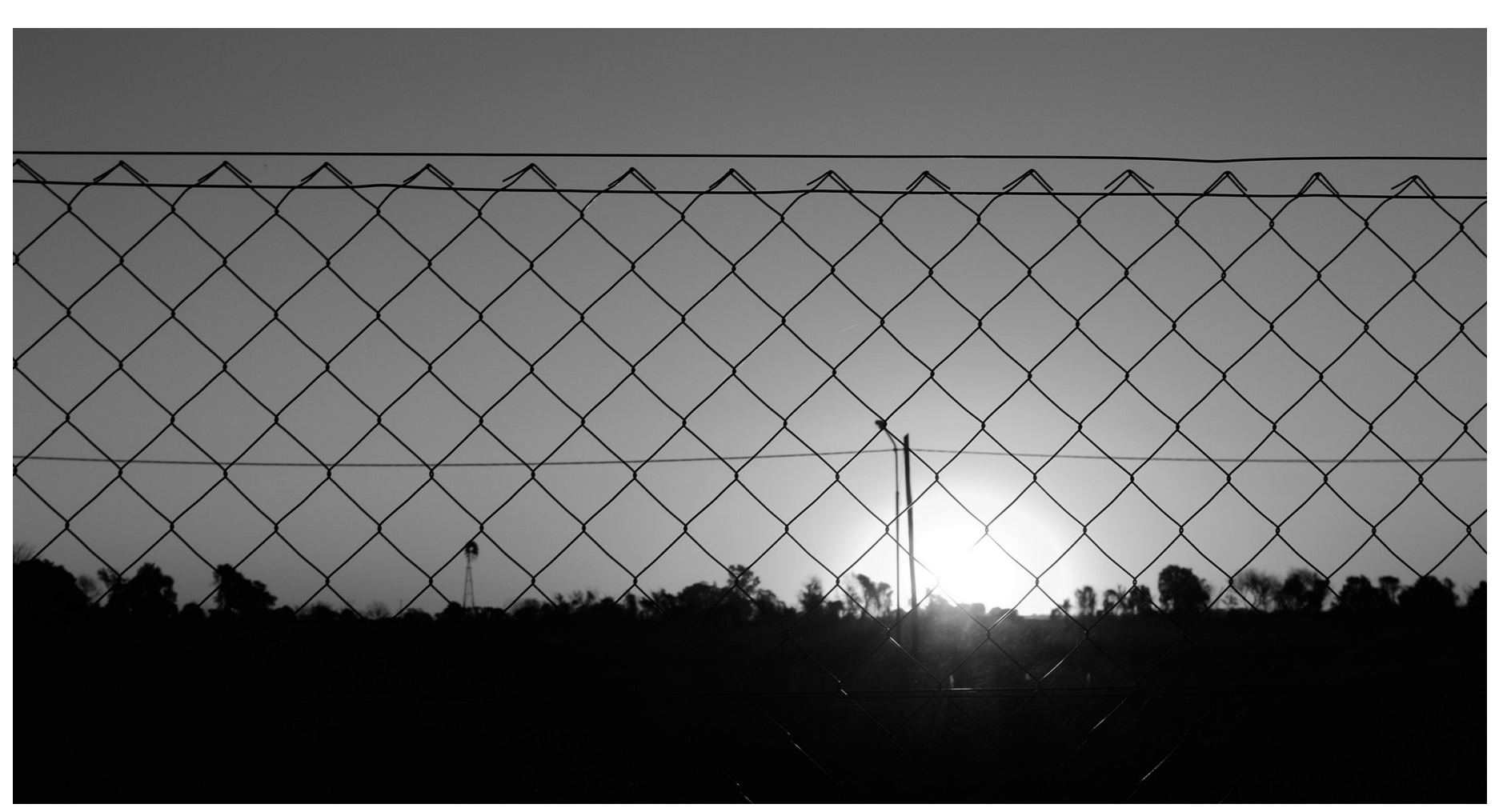

(c) Marcela Pinotti

\section{Referencias bibliográficas}

Alsina, C. (2007). Educación matemática e imaginación. UNIÓN, (11), 9-17.

Camilloni, A. (2013). La inclusión de la educación experiencial en el currículo universitario. En Menéndez, G. y otros, Integración docencia y extensión. Otra forma de enseñar y de aprender (pp. 11-21). Santa Fe: Ed. UNL.

Corbalán, F. (1996). Estrategias utilizadas por los alumnos de secundaria en la resolución de juegos. SUMA, (23), 21-32.

Eisner, E. W. (1998). El ojo Ilustrado. Indagación cualitativa y mejora de la práctica educativa. Barcelona: Paidós.

Font, V. (1994). Motivación y dificultades de aprendizaje en matemáticas. SUMA, (17), 10-16.

Freire, P. (2005). Cartas a quien pretende enseñar. México: Siglo XXI Editores.

Gairín, J. y Muñoz, J. M. (2006). Moviendo fichas hacia el pensamiento matemátiCO. SUMA, (51), 15-29

Gimeno Sacristán, J. y Pérez Gómez, A. (2002). Comprender y transformar la enseñanza (11 a ed.). Madrid: Morata.
Hernández, H.; Kataoka, V. y Silva, M. (2010). El uso de los juegos para la promoción del razonamiento probabilìsdtico. UNIÓN, (24), 69-83.

Instituto Nacional de Tecnologías y Formación del Profesorado (2012). Descartes. Matemáticas interactivas. Recuperado de: http://recursostic.educacion.es/descartes/web/materiales_didacticos/Juegos_en_Matematicas/indice.htm(26/04/2015). http://recursostic.educacion.es/descartes/web/materiales_didacticos/Juegos_en_ Matematicas/indice.htm(26/04/2015)

Muñiz-Rodríguez, L.; Alonso, P. y Rodríguez-Muñiz, L. J. (2014). El uso de los juegos como recurso didáctico para la enseñanza y el aprendizaje de las Matemáticas: estudio de una experiencia innovadora. UNIÓN, (39), 19-33.

OCDE (2005). Informe PISA 2003. Recuperado de: https://www.oecd.org/ pisa/39732493.pdf (16/04/16).

Salvador, A. (2013). El juego como recurso didáctico en el aula de Matemáticas. Recuperado de: http://es.slideshare.net/arilaynes/12juego (15/04/2015).

Juego... I Lovatto, M.; Zanabria, C.; Municoy, M. C.; Alaniz, B. y Huespe, A. I 343 